\title{
Outcomes and expanding indications for robotic retroperitoneal lymph node dissection for testicular cancer
}

\author{
Hailiu Yang ${ }^{1}$, Daisy Obiora ${ }^{1}$, Jeffrey J. Tomaszewski ${ }^{2}$ \\ ${ }^{1}$ Division of Urology, Department of Surgery, Cooper University Hospital, Camden, NJ, USA; ${ }^{2}$ Division of Urology, Department of Surgery, MD \\ Anderson Cancer Center at Cooper, Cooper Medical School of Rowan University, Camden, NJ, USA \\ Contributions: (I) Conception and design: All authors; (II) Administrative support: None; (III) Provision of study materials or patients: None; (IV) \\ Collection and assembly of data: None; (V) Data analysis and interpretation: None; (VI) Manuscript writing: All authors; (VII) Final approval of \\ manuscript: All authors. \\ Correspondence to: Hailiu Yang. Division of Urology, Department of Surgery, Cooper University Hospital, 3 Cooper Plaza Suite 411, Camden, NJ \\ 08103, USA. Email: yang-hailiu@cooperhealth.edu.
}

\begin{abstract}
Treatment of testicular cancer has made significant progress in the past decades in terms of reduction of treatment-associated morbidity and preventing over-treatment. At the forefront of this progression is utilization of the da Vinci robot to perform retroperitoneal lymph node dissections (RPLNDs) via a minimally invasive approach. The robot offers multiple potential advantages such as smaller incisions, improved 3D visualization, more precise dissection, and faster convalescence, leading to its increased usage the past several years. In this chapter, we summarize the recent progress made in robotic surgery for testicular cancer and its potential in the future. Promising preliminary data has also renewed interest in defining the role of primary RPLND in patients with seminoma, potentially sparing patients of the harmful long-term radiation and cisplatin-based chemotherapy. SEMS and PRIMETEST trials are ongoing trials that will provide significant insight into this area and potentially expand the role of robotic RPLND.
\end{abstract}

Keywords: Seminoma; robotic retroperitoneal lymph node dissection (robotic RPLND); post-chemotherapy retroperitoneal lymph node dissection (post-chemotherapy RPLND); metastatic testicular cancer

Submitted Sep 30, 2019. Accepted for publication Feb 24, 2020.

doi: $10.21037 /$ tau.2020.03.14

View this article at: http://dx.doi.org/10.21037/tau.2020.03.14

\section{Introduction}

Testicular cancer is one of the most common malignancies in men age 20 to 40 in the United States (1). Ninetyfive percent of testicular neoplasms are germ cell tumors, which are further sub-classified into seminoma and nonseminomatous germ cell tumors (NSGCT). Treatment of testicular tumor consists of initial orchiectomy followed by either observation, retroperitoneal lymph node dissection (RPLND), external-beam radiation (XRT), or cisplatinbased chemotherapy depending on the tumor pathology, clinical stage, and shared-decision making between the patient and physician (2). RPLND is typically performed as initial treatment for low-stage NSGCT (stage IA, IB, and IIA NSGCT), for residual retroperitoneal mass in the post- chemotherapy setting, or as desperation surgery (3).

Traditionally, RPLNDs are performed via an open approach. The first open RPLND (O-RPLND) was reported over 70 years ago (2). At that time, treatment options for testicular cancer were limited and survival was poor. The paradigm of testicular cancer has since shifted dramatically. Survival among patients with advanced testicular cancer has improved from $5-10 \%$ to $80-90 \%$ with the development of cisplatin-based chemotherapy (2). In 2019, the American Cancer Society expects 9,560 new cases of testicular cancer with only 410 deaths (4). Improvements in cancer-specific survival have shifted the focus of treatment from improving survival to decreasing treatmentrelated side-effects. Cisplatin-based chemotherapy 
regimens have significant short-term and long-term side effects, including renal toxicity, bone marrow suppression, hypogonadism, cardiovascular disease, metabolic syndrome, and secondary malignancies (5-7). As a result, the medical oncology community is constantly looking to expand the role of active surveillance, reduced-dose chemotherapy, and RPLNDs, for which the side effects are mostly limited to the immediate post-operative period.

\section{Minimally invasive RPLND}

For many decades, RPLNDs were performed via an open approach. As surgeons became facile with rapidly expanding advances in minimally invasive surgery, the technology was applied to a broadening array of disease sites. Laparoscopic RPLND (L-RPLND) for testicular cancer was first performed in $1992(8,9)$. Since 1992, there have been numerous reports of L-RPLND in the primary (chemotherapy naïve) setting for stage I NSGCT. Due to a significant learning curve, early experiences in L-RPLND were marred by an increased rate of complications, high rate of open conversion, and variable lymph node (LN) yield (10). There were also concerns regarding the ability of laparoscopy to obtain adequate dissection posterior to the great vessels and control significant bleeding. In a metaanalysis, Rassweiler et al. (10) found a highly variable and unacceptable conversion rate (5.8\% to $13.3 \%$ ), complication rate $(5.6 \%$ to $46.7 \%)$, and percentage of positive LNs (5.8\% to $62.5 \%$ ) for laparoscopic series published from 1994 to 2004. However, as time progressed, so did operator experience-of series performed between 2004 to 2008 involving 499 total patients, there were significant improvements in operative time (mean 204 minutes), complication rates $(15.6 \%)$, conversion rates $(3.8 \%)$, rate of retroperitoneal relapse $(1.4 \%)$, and percentage of positive LNs (25\%), with an average follow-up of 63 months. Laparoscopic series as a whole were able to demonstrate decreased length of stay (LOS) of 3.3 days compared to 6.6 days in O-RPLND series (10). Despite promising initial data revealing more rapid convalescence, recovery, and improved cosmesis with laparoscopy, L-RPLND is technically challenging and requires a steep learning curve, effectively limiting its widespread adoption.

\section{Robotic-assisted laparoscopic RPLND (RA- RPLND)}

About a decade after L-RPLND was reported, the first RA-
RPLND was performed in 2006 (11). Robotic surgery offers several potential advantages compared to its laparoscopic counterpart, including high-definition $3 \mathrm{D}$ visualization, increased freedom of movement, and minimization of tremors. The advantages allow the surgeon to potentially reap the benefits of minimally invasive surgery while overcoming the technical challenges associated with laparoscopic surgery.

RA-RPLND was initially performed in the primary treatment setting for stage I and IIA NSGCT. The current literature reports of RA-RPLND, while limited to retrospective case series, have shown improved convalescence with good oncologic and surgical outcomes among the aforementioned patient population (12-19). In the largest series presented to date, 47 primary (chemotherapy naïve) RA-RPLNDs were performed across 6 institutions in the United States. Among their series of patients with clinical stage I and IIA NSGCT, the median operative time was 235 minutes, with a mean estimated blood loss (EBL) of $40 \mathrm{cc}$, median LOS of 1 day, and overall complication rate of $14 \%$. The median LN yield was 26 and the 2-year recurrence-free survival was $97 \%$, with a median follow-up of 16 months (16). Stephanian et al. (13) presented a single-surgeon series of 20 patients with varying clinical stage and pathology. The median operating time was 293 minutes, with median EBL of 50 cc, and median LOS of 1 day. There was one major complication-a ureteral injury identified intraoperatively and treated with a ureteroureterostomy. The median $\mathrm{LN}$ yield was 19.5 and there no recurrences with a median follow-up of 49 months. These good results in the pre-chemotherapy setting were replicated by several other institutions $(12,19,20)$.

To date, there are no series comparing RA-RPLND to O-RPLND. There is one single retrospective series comparing RA-RPLND to L-RPLND. This single-surgeon series compared the robotic $(n=16)$ and laparoscopic approach $(\mathrm{n}=21)$ to a modified template RPLND for patients with stage I NSGCT (19). Both groups had one major post-operative complication and one conversion to open, suggesting that both approaches are equally safe. While the LN yield was adequate (30 in the RA-RPLND group and 21 in the L-RPLND group), follow-up was inadequate to make any conclusions about recurrence rate.

Post-chemotherapy RPLND is far more technically challenging due to desmoplastic reaction and bulky residual disease in some patients. The improved visualization, minimization of tremors, and increased degree of freedom of the robot makes it the ideal minimally-invasive approach 
Table 1 Outcomes of published post-chemotherapy RA-RPLND series

\begin{tabular}{|c|c|c|c|c|c|c|c|c|c|c|c|}
\hline Study & $\begin{array}{c}\text { Total } \\
\text { patients }\end{array}$ & $\begin{array}{l}\text { Post- } \\
\text { chemo }\end{array}$ & $\begin{array}{l}\text { Follow-up } \\
\text { (mo) }\end{array}$ & $\begin{array}{l}\mathrm{LN} \\
\text { yield }\end{array}$ & $\begin{array}{c}\text { Open } \\
\text { conversion, \% }\end{array}$ & $\begin{array}{c}\text { Major } \\
\text { complication, \% }\end{array}$ & $\begin{array}{l}\text { OR } \\
\text { time }\end{array}$ & LOS & EBL & $\begin{array}{l}\text { Anterograde } \\
\text { ejaculation, \% }\end{array}$ & $\begin{array}{l}\text { In-template } \\
\text { recurrence }\end{array}$ \\
\hline $\begin{array}{l}\text { Cheney } \\
\text { et al. (12) }\end{array}$ & 18 & 8 & 22 & 18 & 25.0 & 0 & 369 & 2.2 & 313 & NR & 0 \\
\hline $\begin{array}{l}\text { Kamel } \\
\text { et al. (17) }\end{array}$ & 12 & 12 & 30 & 12 & 12.5 & 8 & 312 & 3.2 & 475 & 80 & 0 \\
\hline $\begin{array}{l}\text { Singh } \\
\text { et al. (18) }\end{array}$ & 13 & 13 & 23 & 20 & 0 & 31 & 200 & 4.0 & 120 & NR & 0 \\
\hline $\begin{array}{l}\text { Overs } \\
\text { et al. (15) }\end{array}$ & 11 & 11 & 24 & 7 & 0 & 0 & 150 & 3.0 & 120 & 73 & 0 \\
\hline
\end{tabular}

RPLND, retroperitoneal lymph node dissection; LN, lymph node; LOS, length of stay; EBL, estimated blood loss; NR, not reported.

in this setting. In the past decade, post-chemotherapy RARPLND has been attempted by several groups and their outcomes have been acceptable (See Table 1) $(12,15,17,21)$. Cheney et al. (12) reported a series of 17 RA-RPLND cases, including 8 post-chemotherapy RA-RPLNDs. Of these 8 patients, 2 were converted to open due to robotic malfunction and bleeding. Additionally, 3 patients were deemed ineligible for robotic surgery due to bulky disease. Despite the high rate of open conversion, they reported no major complications and no in-template recurrence at a median follow-up of 22 months. Compared to primary RARPLND, post-chemotherapy RA-RPLND had a longer average operating time (369 vs. 311 mins), but comparable EBL (313 vs. $100 \mathrm{cc}$ ), LN yield (18 vs. 22 nodes), and LOS (2.2 vs. 2.7 days). Kamel et al. (17) describe a series of 12 post-chemotherapy RA-RPLND. Eleven of 12 surgeries were completed robotically with an average operative time of 312 minutes. The mean EBL was 475 minutes, and mean LOS 3.2 days. There was one major complication (aortic injury and subsequent pseudoaneurysm) and two minor complications. At a median follow up of 31 months, there were no recurrences. In terms of oncological outcomes, the mean number of LNs excised was only 12. Overs et al. (15) also reported a series of 11 patients who underwent postchemotherapy RA-RPLND. Their median operative time was 153 minutes with median EBL of $120 \mathrm{cc}$ and median LOS of 3 days. In terms of oncological efficacy, Overs et al. only removed an average of $7 \mathrm{LNs}$ and followed the patients for a median of 4 months. During the limited follow-up period, there were no major complications or recurrences. To date, an in-template recurrence has not been reported in any RA-RPLND series.

RA-RPLND has a promising future in testicular cancer. RA-RPLND is a less morbid procedure compared to O-RPLND in select patients. Despite these advantages, the evidence for its oncologic efficacy is not as robust as the evidence for O-RPLND. The length of follow-up is limited in most series to less than 2 years. Additionally, the number of harvested LN's is highly variable (as low as 7) (15). Another concern is that the evidence supporting RA-RPLND is limited to small single-institution series without adequate comparison to L-RPLND or O-RPLND. Thus, generalizability is limited and the evidence may be subject to publication bias. A large multi-institutional study with long-term follow-up and standardized protocols is warranted.

The authors actively maintain a database of robotic RPLND cases performed at our institution. To-date, we have performed 7 RA-RPLNDS by a single high-volume robotic surgeon, 6 of which were performed in the postchemotherapy setting. Full bilateral template dissection was performed in all patients with a median yield of 33 LNs. Our patients had a median EBL of $50 \mathrm{cc}$ and LOS of 2 days. We had 4 (57\%) total complications and 2 (28\%) 


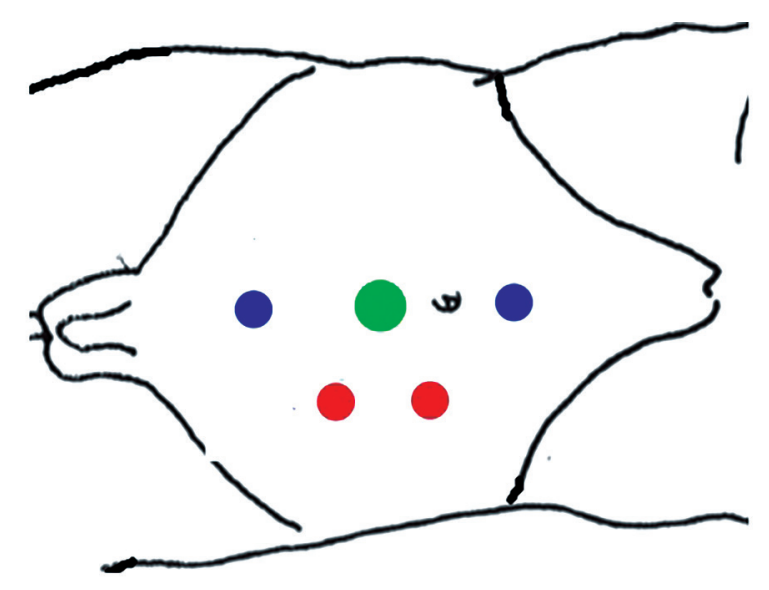

Figure 1 Example of port placement used in the flank transperitoneal approach-used in Cost et al. (14). Green: $12 \mathrm{~mm}$ robot camera port; blue: $8 \mathrm{~mm}$ robot ports; red: assistant ports.

major complications (chylous ascites and thermal injury to the ureter). With a median follow-up of 7 months, we had no in-template recurrences. Of note, we did have 1 open conversion and 3 candidates were deemed ineligible for robotic surgery due to encasement of major vessels or high tumor burden. Our experience illustrates the difficulty of performing post-chemotherapy RA-RPLND and the importance of good patient selection.

\section{Surgical approach}

In general, two approaches to RA-RPLND have been reported in the literature. We will outline the key steps in both of these approaches; step-by-step instructions on these procedures will not be discussed but can be found in the referenced papers. Both approaches have their own limitations and there is inadequate evidence to suggest the superiority of one approach. The da Vinci Xi surgical platform (Intuitive Surgical, Sunnyvale, CA, USA) is able to overcome a lot of the weaknesses of approaches performed using the earlier Si platform. As with O-RPLND, RARPLND should adhere to the same basic surgical principles such as obtaining adequate exposure, performing meticulous dissection, and sparing nerves.

\section{Flank approach}

The flank transperitoneal approach was the initial approach reported by Dr. Davol in 2006 (11). In this approach, the patient is positioned the flank position with the table flexed. A variety of port placements have been reported using this approach; typically, 3-4 robotic ports and 1-2 assistant ports are placed on the side of dissection or midline (Figure 1). The robot is then docked over the patient's shoulder or flank area. Exposure to the retroperitoneum is obtained by incision along the white line of Toldt and reflection of the colon medially. A liver retractor can be employed on the right side, when necessary. This approach allows adequate access to the affected side of the retroperitoneum as well as the spermatic cord. When a bilateral template is required, this approach may require redocking $(12,17)$, though singledocking has been reported (21).

\section{Supine approach}

The supine transperitoneal approach is a popular approach recently reported by various groups $(12,13)$. In this approach, the patient is positioned in the supine position and placed in steep Trendelenburg to allow the bowel to fall cephalad. The robot is then docked over the patient's head (Si system) or side (Xi system) (Figure 2). Robotic ports are placed diagonally in the lower abdomen tilted towards the laterality of the involved testicle. The procedures start with the incision of the posterior peritoneum up to the ligament of Treitz to gain exposure to the retroperitoneum. Using a monofilament suture on a straight needle, the small bowel is suspended to the abdominal wall, and a thorough node dissection is performed using the standard template. On the Si platform, the two major limitations of this approach are the need to dock over the patient's head, and the need to redock to excise the spermatic cord (17). However, on the Davinci Xi platform, the system allows for side docking and dissection of a full bilateral template with excision of the cord remnant without the need for redocking (13).

\section{Expanding role of RPLND}

Given the significant side effects of cisplatin-based chemotherapy, two groups are currently investigating the role of RPLND as an alternative to chemotherapy in the setting of low-volume metastatic seminoma. The results of these two studies will have a significant impact on the utility of RA-RPLND given that seminoma accounts for $52-56 \%$ of germ cell tumors and $15 \%$ have metastatic disease at the time of presentation (2). The advantages of primary RPLND for seminoma are accurate pathologic 


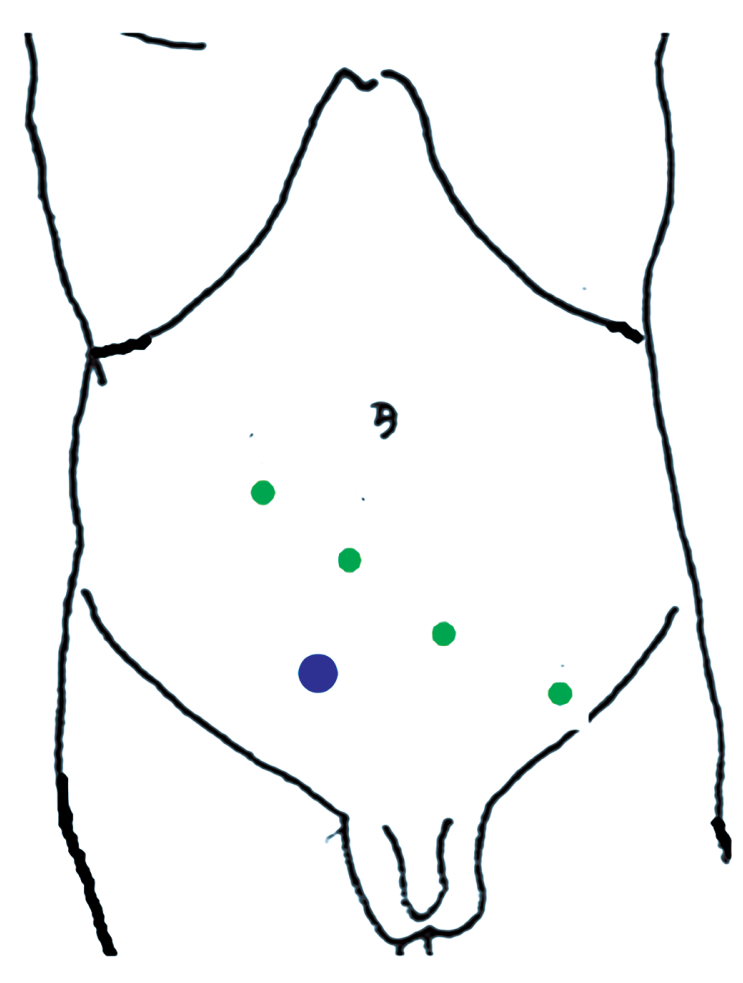

Left testicular tumor

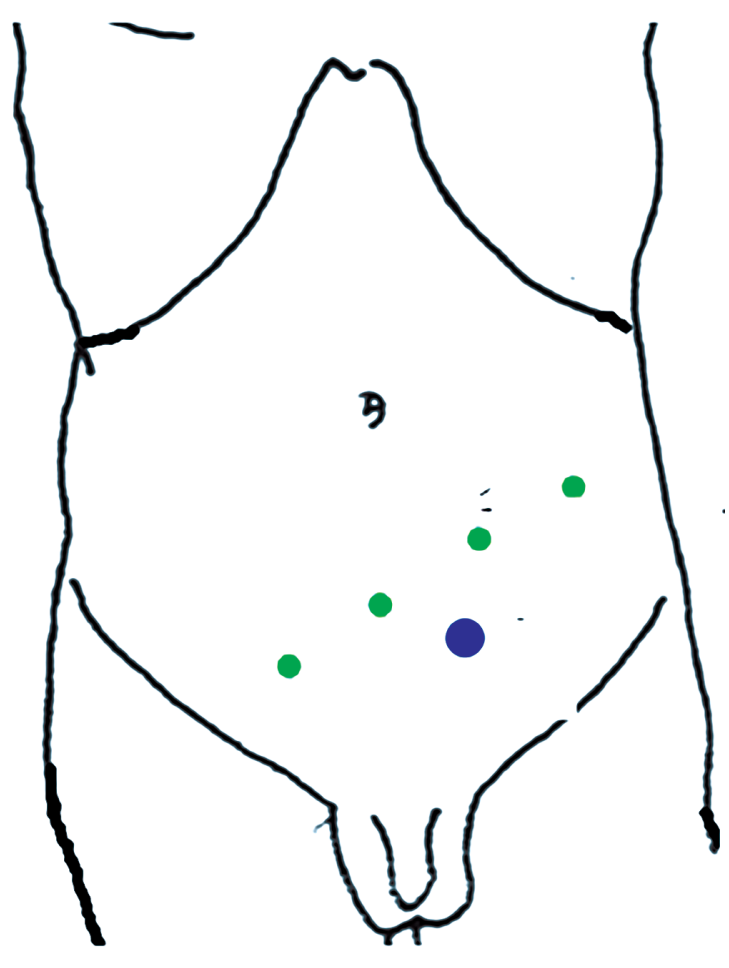

Right testicular tumor

Figure 2 Example of the port placement used in the supine approach—used by the authors. Green: 8 mm Xi robot ports, which can be used for the camera or instruments; blue: $12 \mathrm{~mm}$ AirSeal assistant port.

staging and avoidance of the side effects of chemotherapy and radiation in the majority of patients. Several small series have reported that primary RPLND in seminoma (stage I to IIC) resulted in a significant amount of discordant staging and a low recurrence rate. Warszawski et al. (23) retrospectively compared seminoma patients treated with XRT and full-template RPLND. Of the 63 patients who had primary RPLND, $17.5 \%$ of patients were upstaged and $6.3 \%$ of patients were down-staged. The in-field and overall relapse rate were higher in the RPLND group, $9.5 \%$ vs. $2 \%$ and $14.3 \%$ vs. $9.1 \%$, respectively. However, there were no differences in overall survival and rate of relapse, which occurred predominantly in stage IIB and IIC patients, was low in both groups. Mezvrishvili et al. (24) reported 14 patients who underwent modified-template RPLND for stage I and IIA seminoma. At a median followup of 56 months, there were no major post-operative complications and no patients with disease recurrence. Recently, Hu et al. (25) reported a series of 4 patients who underwent a modified-template RPLND for stage IIA and
IIB seminoma, and there was no evidence of recurrence at a median follow-up of 25 months.

These promising findings have led to renewed interest in primary RPLND in seminoma by investigators in Germany and United States. The SEMS (Surgery for Early Metastatic Seminoma) trial is a prospective phase II study currently recruiting metastatic seminoma patients at 9 institutions across the United States with LN size of $1-3 \mathrm{~cm}$ (26). The PRIMETEST trial (Trial for Primary Retroperitoneal Lymph Node Dissection in Stage II A/B Seminoma Patients Without Adjuvant Treatment) is a prospective multi-center phase II study recruiting patients at multiple institutions in Germany with seminoma metastases less than $5 \mathrm{~cm}$ (27). Several notable differences between the studies include: PRIMETEST allows for 1 dose of carboplatin for stage I disease whereas SEMS does not, and PRIMETEST allows for minimally invasive approaches while SEMS mandates an open approach. If the results of these two trials support primary RPLND in seminoma, the number of RPLNDs performed will drastically increase. Given the advantages of 
robotic surgery, this paradigm shift will likely translate to more RA-RPLNDs.

\section{Conclusions}

Robotic technology continues to improve and establish a presence in urologic oncology. Due to its minimization of tremors, improved visualization, and increased freedom of movement, robotic surgery provides the benefits of minimally invasive surgery without the technical challenges posed by conventional laparoscopy for RPLND. Preliminary evidence suggests that RA-RPLND provides adequate surgical and oncological outcomes. RA-RPLND will likely have a major role in the future of testicular cancer treatment.

\section{Acknowledgments}

Funding: None.

\section{Footnote}

Provenance and Peer Review: This article was commissioned by the editorial office, Translational Andrology and Urology for the series "Controversies in Minimally Invasive Urologic Oncology". The article has undergone external peer review.

Conflicts of Interest: All authors have completed the ICMJE uniform disclosure form (available at http://dx.doi. org/10.21037/tau.2020.03.14). The series "Controversies in Minimally Invasive Urologic Oncology" was commissioned by the editorial office without any funding or sponsorship. Jeffrey J. Tomaszewski served as the unpaid Guest Editor of the series, and he is a consultant for Genomic Health. The authors have no other conflicts of interest to declare.

Ethical Statement: The authors are accountable for all aspects of the work in ensuring that questions related to the accuracy or integrity of any part of the work are appropriately investigated and resolved.

Open Access Statement: This is an Open Access article distributed in accordance with the Creative Commons Attribution-NonCommercial-NoDerivs 4.0 International License (CC BY-NC-ND 4.0), which permits the noncommercial replication and distribution of the article with the strict proviso that no changes or edits are made and the original work is properly cited (including links to both the formal publication through the relevant DOI and the license). See: https://creativecommons.org/licenses/by-nc-nd/4.0/.

\section{References}

1. Horner MJ, Ries LAG, Krapcho M, et al. SEER cancer statistics review, 1975-2006. Bethesda: National Cancer Institute, 2009.

2. Stephenson AJ, Gilligan TD. Neoplasms of the testis. In: Wein AJ, Kavoussi LR, Novick AC, et al. Campbell-Walsh Urology. 10th ed. Philadelphia: Elsevier, 2012:837-70.

3. Daneshmand S, Dorff T. Update Series (2018) Lesson 3: Update on medical and surgical management of advanced testis cancer. In: AUA Update Series. AUA University, 2018.

4. American Cancer Society, Key Statistics for Testicular Cancer. Available online: https://www.cancer.org/cancer/ testicular-cancer/about/key-statistics.htm (Accessed 9/28/2019).

5. Beyer J, Albers P, Altena R, et al. Maintaining success, reducing treatment burden, focusing on survivorship: highlights from the third European consensus conference on diagnosis and treatment of germ-cell cancer. Ann Oncol 2013;24:878-88.

6. Zagars GK, Ballo MT, Lee AK, et al. Mortality after cure of testicular seminoma. J Clin Oncol 2004;22:640-7.

7. Hanks GE, Peters T, Owen J. Seminoma of the testis: long-term beneficial and deleterious results of radiation. Int J Radiat Oncol Biol Phys 1992;24:913-9.

8. Rukstalis DB, Chodak GW. Laparoscopic retroperitoneal lymph node dissection in a patient with stage 1 testicular carcinoma. J Urol 1992;148:1907-9; discussion 1909-10.

9. Hulbert JC, Fraley EE. Laparoscopic retroperitoneal lymphadenectomy: new approach to pathologic staging of clinical stage I germ cell tumors of the testis. J Endourol 1992;6:123-5.

10. Rassweiler JJ, Scheitlin W, Heidenreich A, et al. Laparoscopic retroperitoneal lymph node dissection: does it still have a role in the management of clinical stage I nonseminomatous testis cancer? A European perspective. Eur Urol 2008;54:1004-15.

11. Davol P, Sumfest J, Rukstalis D. Robotic-assisted laparoscopic retroperitoneal lymph node dissection. Urology 2006;67:199.

12. Cheney SM, Andrews PE, Leibovich BC, et al. Robotassisted retroperitoneal lymph node dissection: technique and initial case series of 18 patients. BJU Int 2015;115:114-20. 
13. Stepanian S, Patel M, Porter J. Robot-assisted laparoscopic retroperitoneal lymph node dissection for testicular cancer: evolution of the technique. Eur Urol 2016;70:661-7.

14. Cost NG, DaJusta DG, Granberg CF, et al. Robot-assisted laparoscopic retroperitoneal lymph node dissection in an adolescent population. J Endourol 2012;26:635-40.

15. Overs C, Beauval JB, Mourey L, et al. Robot-assisted post-chemotherapy retroperitoneal lymph node dissection in germ cell tumor: is the single-docking with lateral approach relevant? World J Urol 2018;36:655-61.

16. Pearce SM, Golan S, Gorin MA, et al. Safety and early oncologic effectiveness of primary robotic retroperitoneal lymph node dissection for nonseminomatous germ cell testicular cancer. Eur Urol 2017;71:476-82.

17. Kamel MH, Littlejohn N, Cox M, et al. Postchemotherapy robotic retroperitoneal lymph node dissection: institutional experience. J Endourol 2016;30:510-9.

18. Singh A, Chatterjee S, Bansal P, et al. Robot-assisted retroperitoneal lymph node dissection: feasibility and outcome in postchemotherapy residual mass in testicular cancer. Indian J Urol 2017;33:304-9.

19. Harris KT, Gorin MA, Ball MW, et al. A comparative analysis of robotic vs laparoscopic retroperitoneal lymph node dissection for testicular cancer. BJU Int 2015;116:920-3.

20. Abdul-Muhsin HM, L'esperance JO, Fischer K, et al. Robot-assisted retroperitoneal lymph node dissection in testicular cancer. J Surg Oncol 2015;112:736-40.

Cite this article as: Yang H, Obiora D, Tomaszewski JJ. Outcomes and expanding indications for robotic retroperitoneal lymph node dissection for testicular cancer. Transl Androl Urol 2021;10(5):2188-2194. doi: 10.21037/tau.2020.03.14
21. Stout TE, Soni SD, Goh AC. Post-chemotherapy robotic bilateral retroperitoneal lymph node dissection using a novel single-dock technique. J Robot Surg 2016;10:353-6.

22. Tamhankar AS, Patil SR, Ojha SP, et al. Therapeutic supine robotic retroperitoneal lymph node dissection for post-chemotherapy residual masses in testicular cancer: technique and outcome analysis of initial experience. J Robot Surg 2019;13:747-56.

23. Warszawski N, Schmücking M. Relapses in earlystage testicular seminoma: radiation therapy versus retroperitoneal lymphadenectomy. Scand J Urol Nephrol 1997;31:355-9.

24. Mezvrishvili Z, Managadze L. Retroperitoneal lymph node dissection for high-risk stage I and stage IIA seminoma. Int Urol Nephrol 2006;38:615-9.

25. Hu B, Shah S, Shojaei S, et al. Retroperitoneal lymph node dissection as first-line treatment of node-positive seminoma. Clin Genitourin Cancer 2015;13:e265-9.

26. Retroperitoneal lymph node dissection in treating patients with testicular seminoma. Available online: https:// clinicaltrials.gov/ct2/show/NCT02537548 (Accessed 9/28/2019).

27. Trial to evaluate progression free survival with primary retroperitoneal lymph-node dissection (pRPLND) only in patients with seminomatous testicular germ cell tumors with clinical stage IIA/B (PRIMETEST). Available online: https://clinicaltrials.gov/ct2/show/NCT02797626 (Accessed 9/28/2019). 